\title{
A New Approach for Recognition of Mosaic Textures by LBP Based On RGB Model
}

\author{
L.Sumalatha and B.Sujatha \\ ${ }^{1}$ Department of Computer Science \& Engg., UCEK, JNTUK Kakinada \\ sumalatha.lingamgunta@gmail.com \\ ${ }^{2}$ Department of Computer Science \& Engg., GIET, Rajahmundry \\ birudusujatha@gmail.com
}

\begin{abstract}
The method is based on recognizing that certain local binary patterns, termed are fundamental properties of local image texture and their occurrence histogram is proven to be a very powerful texture feature. The Local Binary Pattern (LBP) is a texture descriptor based on the probability of occurrence of elementary binary patterns (texels) defined over a circular window. A new feature set derived from the LBP, called the LBP-Constant-Symmetry (LBP-CS) and LBP-High-Symmetry (LBP-HS) are proposed for recognition of stone textures. The features are computed from each band of an isotropic color LBP Matrix for recognition. The tests were conducted in a variety of industrial samples. The obtained results are promising and show the possibility of efficiently recognizing complex industrial products based on color and texture features
\end{abstract}

\section{KEYWORDS}

Local binary patterns, Histogram, LBP-CS, LBP-HS

\section{INTRODUCTION}

The Local Binary Pattern (LBP) approach has evolved to represent a significant breakthrough in texture analysis, outperforming earlier methods in many applications. Perhaps the most important property of the LBP operator in real-world applications is its tolerance against illumination changes. Another equally important is its computational simplicity, which makes it possible to analyze images in challenging real-time settings. Based on LBP approach the present study proposes a new method for the analysis of color stone textures. For this the present study evaluated co-occurrence features on patterns extracted from local binary operator. The present study also derived new statistical parameters called LBP constant and high symmetry. These parameters are also used in the color stone texture analysis.

Image texture analysis is an important fundamental problem in computer vision. During the past few years, several authors have developed theoretically and computationally simple, but very efficient nonparametric methodology for texture analysis based on LBP $[1,2,3,4,5,6,7,8,9]$. The LBP texture analysis operator is defined as a gray scale invariant texture measure, derived from a general definition of texture in a local neighbourhood. For each pixel in an image, a binary code is produced by thresholding its value with the value of the centre pixel. A histogram is created to collect up the occurrences of different binary patterns. The basic version of the LBP operator considers only the eight neighbours of a pixel, but the definition has been extended to include all circular neighbourhoods with any number of pixels $[10,11,12]$. Through its extensions, the LBP operator has been made into a really powerful measure of image texture, showing excellent results in terms of accuracy and computational complexity in many empirical studies. The LBP operator can be seen as a unifying approach to the traditionally divergent 
statistical and structural models of texture analysis. Perhaps the most important property of the LBP operator in real world applications is its tolerance against illumination changes. Another equally important is its computational simplicity, which makes it possible to analyze images in challenging real-time settings.

The LBP method has already been used in a large number of applications all over the world, including visual inspection, image retrieval, remote sensing, biomedical image analysis, face image analysis, motion analysis, environment modelling, and outdoor scene analysis. The present paper developed co-occurrence features on LBP and also developed a new statistical parameter on LBP for the recognition of stone textures.

\section{REPRESENTATION OF LBP}

The present section introduces the basic concept of LBP .It is a gray-scale invariant texture measure computed from the analysis of a $3 \times 3$ local neighbourhood over a central pixel. The LBP is based on a binary code describing the local texture pattern. This code is built by thresholding a local neighbourhood by the gray value of its centre.

In a square-raster digital image, each pixel is surrounded by eight neighbouring pixels. The local texture information for a pixel can be extracted from a neighbourhood of $3 \times 3$ pixels, which represents the smallest complete unit (in the sense of having eight directions surrounding the pixel). A neighbourhood of $3 \times 3$ pixels is denoted by a set containing nine elements: $P=\left\{P_{0}, P_{1}\right.$

$\left.\ldots P_{8}\right\}$, here $\mathrm{P}_{0}$ represents the intensity value of the central pixel and $\mathrm{P}_{i}\{\mathrm{i}=1,2 \ldots 8\}$, is the intensity value of the neighbouring pixel $i$. The eight neighbours are labelled using a binary code $\{0,1\}$ obtained by comparing their values to the central pixel value. If the tested gray value is below the gray value of the central pixel, then it is labelled 0 , otherwise it is assigned the value 1 as described by the Equation 1.

$$
p_{i}^{\prime}=\left\{\begin{array}{cc}
0 & \text { if } I\left(x_{i}, y_{i}\right)<I\left(x_{0}, y_{0}\right) \\
1 & \text { otherwise }
\end{array}\right.
$$

$\mathrm{P}_{i}^{\prime}$ is the obtained binary code, $P_{i}$ is the original pixel value at position $i$ and $P$ is the central pixel value. The Fig. 1(a) shows the grey level values of a $3 \times 3$ neighbourhood of an image. And the Fig. 1(b) shows its corresponding binary labelling based on Equation 1. The binary weights of the given $3 \times 3$ neighbourhood are calculated by the Equation 2 .

$$
\text { Weights }_{L B P}=\sum_{i=0}^{i=7} P_{i} \times 2^{i-1}
$$

As each element of LBP has one of the two possible values, the combination of all the eight elements results in $2^{8}=256$ possible local binary patterns ranging from 0 to 255 . There is no unique way to label and order the 255 LBP on a $3 \times 3$ neighbourhood. 
Signal \& Image Processing : An International Journal (SIPIJ) Vol.4, No.1, February 2013

\begin{tabular}{|l|l|l|}
\hline 63 & 28 & 45 \\
\hline 88 & $\mathbf{4 0}$ & 35 \\
\hline 67 & 40 & 21 \\
\hline
\end{tabular}

(a)

\begin{tabular}{|l|l|l|}
\hline $2^{0}$ & $2^{1}$ & $2^{2}$ \\
\hline $2^{7}$ & & $2^{3}$ \\
\hline $2^{6}$ & $2^{5}$ & $2^{4}$ \\
\hline
\end{tabular}

(d)

\begin{tabular}{|l|l|l|}
\hline 1 & 0 & 1 \\
\hline 1 & & 0 \\
\hline 1 & 1 & 0 \\
\hline
\end{tabular}

(b)

\begin{tabular}{|c|c|c|}
\hline 1 & 0 & 2 \\
\hline 128 & & 0 \\
\hline 64 & 32 & 0 \\
\hline
\end{tabular}

(d)

Fig. 1 (a) Sample Gray level Neighbourhood (b) Conversion of Gray level to Binary Neighbourhood (c) Representation of binary Weights (d) Represented Values with Binary Weights.

Fig. 1 shows an example on how to compute LBP. The original $3 \times 3$ neighbourhood is given in Fig. 1(a). The central pixel value is used as a threshold in order to assign a binary value to its neighbours. Fig. 1(b) shows the result of thresholding the $3 \times 3$ neighbourhood. The obtained values are multiplied by their corresponding weights as shown by Fig. 1(c). The result is given in Fig. 1(d). The sum of the resulting values gives the LBP measure which is 227 in this case the central pixel 40 is replaced by the obtained LBP value 227. A new LBP image is constructed by processing each pixel and its $3 \times 3$ neighbours in the original image. The binary weights of Fig. 1 (c) can be given in eight different ways as shown below.

\begin{tabular}{|l|l|l|}
\hline $2^{0}$ & $2^{1}$ & $2^{2}$ \\
\hline $2^{7}$ & & $2^{3}$ \\
\hline $2^{6}$ & $2^{5}$ & $2^{4}$ \\
\hline
\end{tabular}

(a)

\begin{tabular}{|l|l|l|}
\hline $2^{6}$ & $2^{7}$ & $2^{0}$ \\
\hline $2^{5}$ & & $2^{1}$ \\
\hline $2^{4}$ & $2^{3}$ & $2^{2}$ \\
\hline
\end{tabular}

(c)

\begin{tabular}{|l|l|l|}
\hline $2^{4}$ & $2^{5}$ & $2^{6}$ \\
\hline $2^{3}$ & & $2^{7}$ \\
\hline $2^{2}$ & $2^{1}$ & $2^{0}$ \\
\hline
\end{tabular}

(e)

\begin{tabular}{|l|l|l|}
\hline $2^{2}$ & $2^{3}$ & $2^{4}$ \\
\hline $2^{1}$ & & $2^{5}$ \\
\hline $2^{0}$ & $2^{7}$ & $2^{6}$ \\
\hline
\end{tabular}

(g)

\begin{tabular}{|l|l|l|}
\hline $2^{7}$ & $2^{0}$ & $2^{1}$ \\
\hline $2^{6}$ & & $2^{2}$ \\
\hline $2^{5}$ & $2^{4}$ & $2^{3}$ \\
\hline
\end{tabular}

(b)

\begin{tabular}{|l|l|l|}
\hline $2^{5}$ & $2^{6}$ & $2^{7}$ \\
\hline $2^{4}$ & & $2^{0}$ \\
\hline $2^{3}$ & $2^{2}$ & $2^{1}$ \\
\hline
\end{tabular}

(d)

\begin{tabular}{|l|l|l|}
\hline $2^{3}$ & $2^{4}$ & $2^{5}$ \\
\hline $2^{2}$ & & $2^{6}$ \\
\hline $2^{1}$ & $2^{0}$ & $2^{7}$ \\
\hline
\end{tabular}

(f)

\begin{tabular}{|l|l|l|}
\hline $2^{1}$ & $2^{2}$ & $2^{3}$ \\
\hline $2^{0}$ & & $2^{4}$ \\
\hline $2^{7}$ & $2^{6}$ & $2^{5}$ \\
\hline
\end{tabular}

(h)

Fig. 2 Eight Different Ways of Calculating LBP on a $3 \times 3$ Neighbourhood.

The value of the LBP changes by the representation of the weights. The LBP can be calculated in 8 different ways for a $3 \times 3$ neighbourhood is shown in Fig. 2 . That is for any $3 \times 3$ neighbourhood one can generate eight LBP values. The LBP value for the Fig.1(a) in all eight directions as represented in Fig.2 is given as 227, 242, 121, 188, 94, 47, 151, and 203 respectively. 
Signal \& Image Processing : An International Journal (SIPIJ) Vol.4, No.1, February 2013

\section{NOVEL SCHEME OF TEXTURE RECOGNITION BASED ON LBP APPROACH ON COLOR SPACE}

\subsection{Color Images}

In the present study LBP is extended to color images. Color images can be represented in different color spaces. The color model is an abstract mathematical model for color representation as vector of numbers. Many color spaces are used in Computer vision. There are basically three color spaces: RGB, HSL and La*b* $[13,14,16]$. RGB color space is the classical color model for color image representation. It uses an additive color mixing model of red, green and blue colors. This color image model is provided directly by the cameras. HSL color model represent the hue, saturation and luminance obtained from color images. HSL is obtained from RGB using color conversion equations $[13,15,16]$. This model is widely used in computer vision for color processing. La*b* color model is a standard color space designed by International Commission on illumination. It is perceptually uniform, and its L component closely matches human perception of lightness. This model is usually as a reference for color difference computation [13, $15,16]$.

The present study applied LBP for RGB color space. In RGB color space LBP is applied for each band. The resultant LBP for each color band for Brick, Granite, Marble and Mosaic textures is displayed in Fig. 4, 5, 6 and 7 respectively. The histogram of each texture in RGB sub bands of LBP is displayed in Fig. 4, 5, 6 and 7 for Brick, Granite, Marble and Mosaic respectively. The histograms clearly reveals that the Granite textures are having high contrast and mosaic textures are having low contrast compared to remaining class of stone textures. Mosaic textures are having equal amount of bright and dark parts in it. The proposed frame work for color texture classification is given in the Fig. 3.

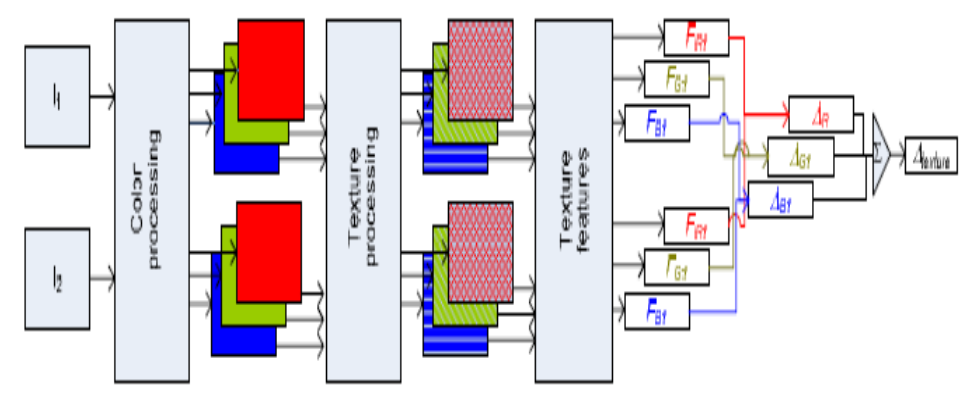

Fig. 3 Frame work for Color-Texture Classification \& Recognition.

The present study attempted an in depth study of LBP on color stone textures. For this LBP scheme is applied on individual sub bands of red, green and blue stone textures. The resultant RGB stone textures are displayed individually, with histograms from Fig. 4 to 7 . The histograms clearly indicate that there is not even slightest difference on brightness or intensity levels of individual color band stone textures using LBP. More over the mean of each individual band of stone textures differed only by a value of one or two. From this, the present study concludes that one need not necessarily experiment on each sub band of stone texture for any analysis or recognition, to analyze or classify or to recognize one can consider only one sub band of color images using LBP. 
Signal \& Image Processing : An International Journal (SIPIJ) Vol.4, No.1, February 2013

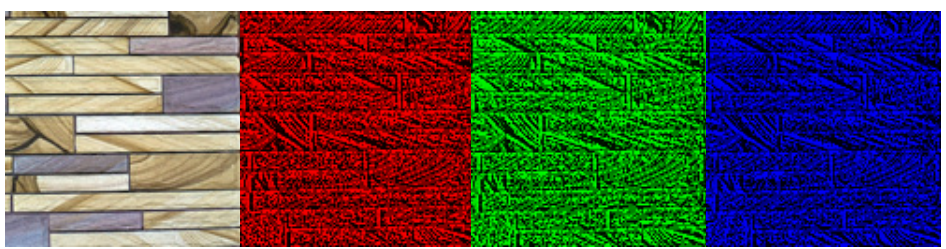

(a)

(b)

(c)

(d)

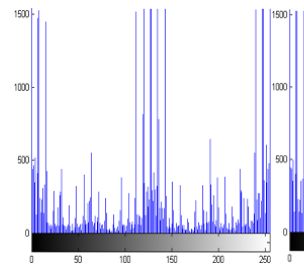

(e)

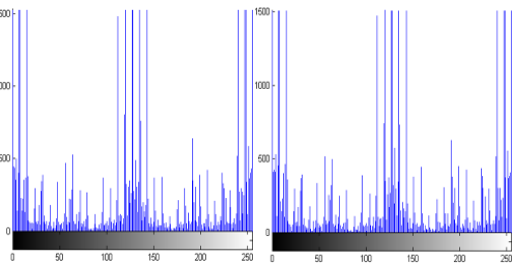

(f)

(g)

Fig. 4 (a) Original Image of Brk2 (b) LBP Result of Red Component (c) LBP Result of Green Component (d) LBP Result of Blue Component (e) Histogram of LBP Resultant Red Component (f) Histogram of LBP Resultant Green Component (g) Histogram of LBP Resultant Blue Component.

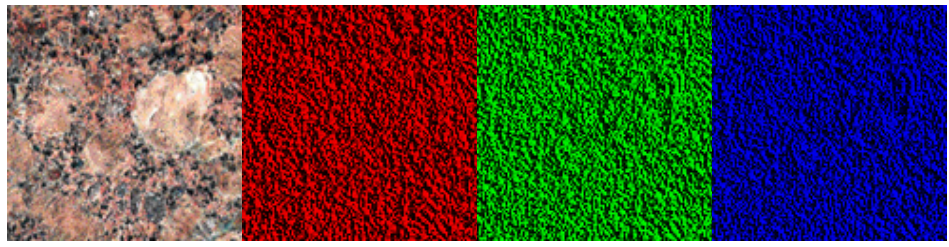

(a)

(b)

(c)

(d)

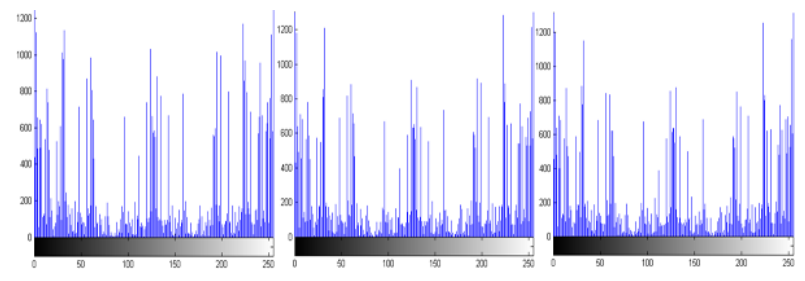

(e)

(f)

(g)

Fig. 5 (a) Original Image of Grn2 (b) LBP Result of Red Component (d) LBP Result of Green Component (f) LBP Result of Blue Component (c) Histogram of LBP Resultant Red Component (e) Histogram of LBP Resultant Green Component (g) Histogram of LBP Resultant Blue Component.

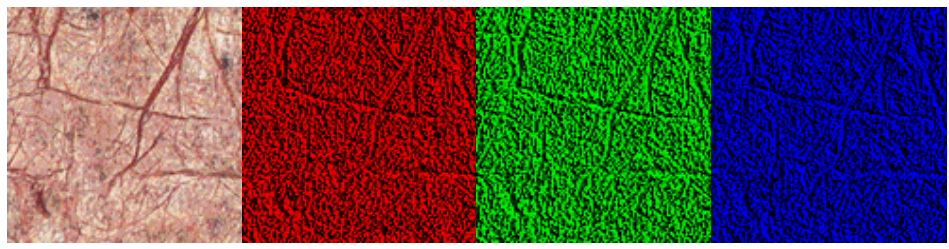


(a)

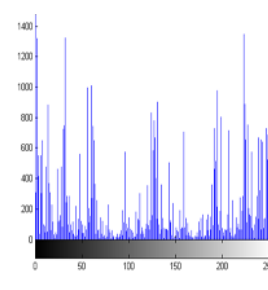

(b)

(e)

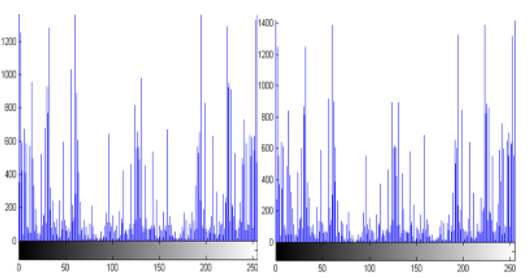

(f) (c)

(g) (d)

Fig. 6 (a) Original Image of Mrb2 (b) LBP Result of Red Component (c) LBP Result of Green Component (d) LBP Result of Blue Component (e) Histogram of LBP Resultant Red Component (f) Histogram of LBP Resultant Green Component (g) Histogram of LBP Resultant Blue Component.

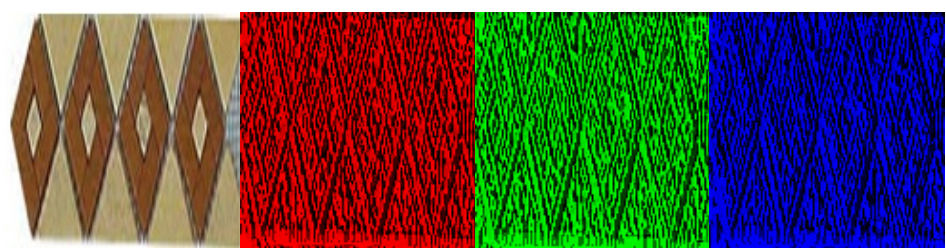

(a)

(b)

(c)

(d)

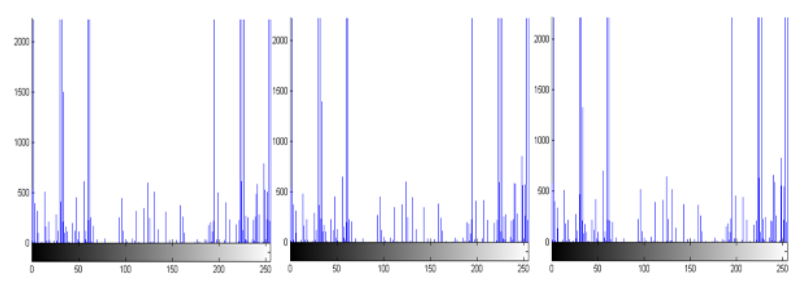

(e)

(f)

(g)

Fig. 7 (a) Original Image of Msa2 (b) LBP Result of Red Component (c) LBP Result of Green Component (d) LBP Result of Blue Component (e) Histogram of LBP Resultant Red Component (f) Histogram of LBP resultant Green Component (g) Histogram of LBP Resultant Blue Component.

\section{RESULTS AND DISCUSSIONS}

In order to recognize mosaic textures, the proposed LBP parameters are computed on each of color bands separately for all stone textures. The average value of each statistical parameter for each class of stone texture on Red, Green and Blue component is listed in the Tables 1, 2, 3 and 4 for Brick, Granite, Marble and Mosaic textures respectively. The tables also contain RGB average value for each statistical parameter for each class of texture. From the Tables 1, 2, 3 and 4, it is clearly evident that all texture features considered show more or less same values for the individual bands of Red, Green and Blue components of stone textures. This factor is true for all stone textures considered. 
Signal \& Image Processing : An International Journal (SIPIJ) Vol.4, No.1, February 2013

Table 1. Average Values on Individual Band of Brick Textures Based on Color LBP using RGB Space

\begin{tabular}{|c|c|c|c|c|c|c|c|}
\hline Texture & Entropy & Energy & Contrast & Homogeneity & Correlation & $\begin{array}{c}\text { LBP- } \\
\text { CS }\end{array}$ & $\begin{array}{c}\text { LBP- } \\
\text { HS }\end{array}$ \\
\hline Red & 11042500.00 & 416000000.00 & 5647075000.00 & 52342.88 & 1.00 & 46.58 & 48.90 \\
\hline Green & 11031750.00 & 416000000.00 & 5636000000.00 & 52025.63 & 1.00 & 47.03 & 49.27 \\
\hline Blue & 11159000.00 & 423000000.00 & 5704525000.00 & 52397.38 & 1.00 & 46.75 & 49.10 \\
\hline Average & 11077750.00 & 418000000.00 & 5662533333.00 & 52255.29 & 1.00 & 46.79 & 49.09 \\
\hline
\end{tabular}

Table 2. Average Values on Individual Band of Granite Textures Based on Color LBP using RGB Space

\begin{tabular}{|c|c|c|c|c|c|c|c|}
\hline Texture & Entropy & Energy & Contrast & Homogeneity & Correlation & $\begin{array}{c}\text { LBP- } \\
\text { CS }\end{array}$ & $\begin{array}{c}\text { LBP- } \\
\text { HS }\end{array}$ \\
\hline Red & 11051375.00 & 422000000.00 & 5584562500.00 & 51971.00 & 1.00 & 46.69 & 48.78 \\
\hline Green & 11181500.00 & 429000000.00 & 5647962500.00 & 52335.75 & 1.00 & 46.65 & 48.59 \\
\hline Blue & 11105125.00 & 425000000.00 & 5621800000.00 & 51322.38 & 1.00 & 46.84 & 49.07 \\
\hline Average & 11112667.00 & 425000000.00 & 5618108333.00 & 51876.38 & 1.00 & 46.73 & 48.81 \\
\hline
\end{tabular}

Table 3. Average Values on Individual Band of Marble Textures Based on Color LBP using RGB Space

\begin{tabular}{|c|c|c|c|c|c|c|c|}
\hline Texture & Entropy & Energy & Contrast & Homogeneity & Correlation & $\begin{array}{c}\text { LBP- } \\
\text { CS }\end{array}$ & $\begin{array}{c}\text { LBP- } \\
\text { HS }\end{array}$ \\
\hline Red & 13346750.00 & 538000000.00 & 6661000000.00 & 61925.00 & 1.00 & 47.00 & 48.58 \\
\hline Green & 12282625.00 & 483000000.00 & 6121225000.00 & 58307.00 & 1.00 & 46.45 & 49.04 \\
\hline Blue & 12333125.00 & 485000000.00 & 6144587500.00 & 59035.63 & 1.00 & 46.61 & 49.09 \\
\hline Average & 12654167.00 & 502000000.00 & 6308937500.00 & 59755.88 & 1.00 & 46.68 & 48.90 \\
\hline
\end{tabular}

Table 4. Average Values on Individual Band of Mosaic Textures Based on Color LBP using RGB Space

\begin{tabular}{|c|c|c|c|c|c|c|c|}
\hline Texture & Entropy & Energy & Contrast & $\begin{array}{c}\text { Homogene } \\
\text { ity }\end{array}$ & $\begin{array}{c}\text { Correlati } \\
\text { on }\end{array}$ & $\begin{array}{c}\text { LBP- } \\
\text { CS }\end{array}$ & $\begin{array}{c}\text { LBP- } \\
\text { HS }\end{array}$ \\
\hline Red & $\begin{array}{c}11427500 \\
00\end{array}$ & $\begin{array}{c}436000000.0 \\
0\end{array}$ & $\begin{array}{c}5835562500 \\
00\end{array}$ & 52903.88 & 1.00 & 37.37 & 30.94 \\
\hline Green & $\begin{array}{c}11474125 \\
00\end{array}$ & $\begin{array}{c}438000000.0 \\
0\end{array}$ & $\begin{array}{c}5841637500 \\
00\end{array}$ & 52802.63 & 1.00 & 37.62 & 30.21 \\
\hline Blue & $\begin{array}{c}11469125 \\
00\end{array}$ & $\begin{array}{c}438000000.0 \\
0\end{array}$ & $\begin{array}{c}5864000000 \\
00\end{array}$ & 52999.88 & 1.00 & 36.56 & 30.99 \\
\hline Average & $\begin{array}{c}11456917 \\
00\end{array}$ & $\begin{array}{c}437000000.0 \\
0\end{array}$ & $\begin{array}{c}5847066667 \\
00\end{array}$ & 52902.13 & 1.00 & 37.18 & 30.71 \\
\hline
\end{tabular}

In order to recognize mosaic textures, the following algorithm is used as follows.

\section{Begin}

1. Extract color bands from a color image (R,G,B or $\mathrm{H}, \mathrm{S}, \mathrm{L}$ or $\left.\mathrm{L}, \mathrm{a}^{*}, \mathrm{~b}^{*}\right)$

2. Apply the texture analysis technique to each band.

3. Compute the texture features $F_{i}$ for each processed color band. 
Signal \& Image Processing : An International Journal (SIPIJ) Vol.4, No.1, February 2013

4. Compute the Euclidian distance between the same features in similar bands of two different images as given in Equation 3 :

$$
\Delta=\sum\left(f_{i}-f_{j}\right)^{2}
$$

5. The final distance is the sum of the three bands distances.

End

In this algorithm, the proposed two statistical features LBP-CS and LBP-HS are considered as given in Equations

LBP-Constant-Symmetry (LBP-CS):

$$
L B P-C S=100 * 1-\frac{\sum_{i=0}^{127} A b s\left[s_{i}-s_{128+i}\right]}{\sum_{i=0}^{255} s_{i}}
$$

LBP-High-Symmetry (LBP-HS):

$$
L B P-H S=100 * 1-\frac{\sum_{i=0}^{127} A b s\left[s_{i}-s_{255-i}\right]}{\sum_{i=0}^{255} s_{i}}
$$

where $S_{i}$ is frequency of occurrence of the LBP value $\mathrm{i}$, in the texture image.

The distance is obtained by computing the Euclidean distance between the above proposed features between the sample texture and database texture. The percentage of recognition rates are listed in Table 5.

Table 5. The Successful Recognition Rate Based on Proposed Color LBP Scheme with LBP Symmetries for Stone Textures

\begin{tabular}{|c|c|}
\hline Texture Class & Successful Recognition Rate \\
\hline Brick & 22.25 \\
\hline Granite & 26.85 \\
\hline Marble & 31.85 \\
\hline Mosaic & 92.45 \\
\hline
\end{tabular}

\section{CONCLUSIONS}

The present paper extended the concept of LBP on color textures. The proposed LBP parameters have shown a high recognition rate of $92 \%$ for Mosaic textures but failed in recognizing the other 
Signal \& Image Processing : An International Journal (SIPIJ) Vol.4, No.1, February 2013

textures. From this, the present study concludes that the proposed features on color LBP lead to a good recognition rate for Mosaic textures only. This is also evident from the histograms of Fig. 4, 5, 6 and 7. From this, the present study concludes that it's not necessary to experiment on each sub band of stone textures for any analysis or recognition.

\section{Acknowledgements}

The authors would like to express their cordial thanks to Dr. K.V.V. Satya Narayana Raju, MLC and Chairman for providing facility to work at the advanced labs of Srinivasa Ramanujan Research Forum (SRRF)-GIET and Sri. K. Sasi Kiran Varma, Managing Director, Chaitanya Group of Institutions for providing moral support and encouragement towards research.

\section{REFERENCES}

[1] Ahonen T., Hadid A. and Pietikainen M. "Face Recognition with Local Binary Patterns," Computer Vision, ECCV Proceedings, pp. 469-481, 2004.

[2] Ahonen T., Pietikainen M., Hadid A. and Maenpaa T. "Face Recognition Based on the Appearance of Local Regions," 17th International Conference on Pattern Recognition III: pp. 153-156, 2004.

[3] Challappa R., Kashyap R.L. and Manjunath B.S. "Model-based Texture Segmentation and Classification," in Handbook of Pattern Recognition and Computer Vision, World Scientific, Teaneck, pp. 277-310, 1993.

[4] Feng X., Pietikainen M. and Hadid A. "Facial Expression Recognition with Local Binary Patterns and Linear Programming," Pattern Recognition and Image Analysis 15 pp. 550-552, 2005.

[5] Hadid A., Pietikainen M. and Ahonen T. "A Discriminative Feature Space for Detecting and Recognizing Faces," IEEE Conference on Computer Vision and Pattern Recognition II: pp. 797-804, 2004.

[6] Heikkila M., Pietikainen M. and Heikkila J. "A Texture-Based Method for Detecting Moving Objects," The 15th British Machine Vision Conference I: pp. 187-196, 2004.

[7] Suresh A and Vijaya Kumar V. et al. "Shape extraction on stone textures based on morphological skeleton approach," communicated to international journal of signal and image processing.

[8] Turtinen M. and Pietikainen M. "Visual Training and Classification of Textured Scene Images," 3rd International Workshop on Texture Analysis and Synthesis pp. 101-106, 2003.

[9] Z. Guo, L. Zhang, D. Zhang, A completed modeling of local binary pattern operator for texture classification, IEEE Transactions on Image Processing 19, 1657-1663, 2010.

[10] Maenpaa T. and Pietikainen, M. "Texture Analysis with Local Binary Patterns," Handbook of Pattern Recognition and Computer Vision, 3rd edn. World Scientific pp. 197-216, 2005.

[11] Ojala T., Pietikainen M., Harwood D. "A Comparative Study of Texture Measures with Classification Based on Feature Distributions. Pattern Recognition," pp. 51-59, 1996.

[12] Ojala T., Pietikainen M., Maenpaa T. "Multiresolution Gray-Scale and Rotation Invariant Texture Classification with Local Binary Patterns," IEEE Transactions on Pattern Analysis and Machine Intelligence 24 pp. 971-987, 2002.

[13] Rafael C. Gonzalez and Richard E. Woods "Digital Image Processing," 2nd ed., Pearson Education (Singapore) Pte. Ltd, Indian Branch, 2003.

[14] Suresh A and Vijaya Kumar V. et al. "Novel methods for stone texture Classification and recognition using linear wavelets' Communicated to Journal of Theoretical and Applied Information Technology.

[15] Tsai, A., Yezzi, A. and Willsky, A. S.,"Curve evolution implementation of the Mumford-Shah functional for image segmentation, de noising, interpolation, and magnification", IEEE Trans. Image Process., vol. 10, pp. 1169-1186, 2001.

[16] Westland S. and Ripamonti A. “Computational Color Science,” John Wiley, 2004. 